Variations tibétaines, Et autres...

\title{
MAY Timothy, The Mongol art of War. Chinggis khan and the Mongol Military System
}

Yardley, Westholme, 2007, xiv + 214 pages, 7 fig., 5 cartes et 23 ill. h-t, index

Abbès Zouache

\section{(2) OpenEdition}

Journals

Édition électronique

URL : https://journals.openedition.org/emscat/1916

DOI : 10.4000/emscat.1916

ISSN : 2101-0013

Éditeur

Centre d'Etudes Mongoles \& Sibériennes / École Pratique des Hautes Études

Référence électronique

Abbès Zouache, "MAY Timothy, The Mongol art of War. Chinggis khan and the Mongol Military System », Études mongoles et sibériennes, centrasiatiques et tibétaines [En ligne], 42 | 2011, mis en ligne le 20 décembre 2011, consulté le 13 juillet 2021. URL : http://journals.openedition.org/emscat/1916 ; DOI https://doi.org/10.4000/emscat.1916

Ce document a été généré automatiquement le 13 juillet 2021.

(c) Tous droits réservés 


\section{MAY Timothy, The Mongol art of War. Chinggis khan and the Mongol Military System \\ Yardley, Westholme, 2007, xiv + 214 pages, 7 fig., 5 cartes et 23 ill. h-t, index}

\section{Abbès Zouache}

\section{RÉFÉRENCE}

MAY Timothy, The Mongol art of War. Chinggis khan and the Mongol Military System, Yardley, Westholme, 2007, xiv + 214 pages, 7 fig., 5 cartes et 23 ill. h-t, index

1 Assurément, le titre est signe ${ }^{1}$. Celui de l'ouvrage (en partie tiré de sa thèse de doctorat ${ }^{2}$ ) - publié par Timothy May est on ne peut plus explicite : il y eut bien un « art de la guerre » et un «système militaire " propres aux Mongols, qui ne formaient pas les hordes armées indisciplinées et adeptes d'une violence désincarnée que si souvent l'on s'est plu à décrire. Non qu'une telle image soit toujours prégnante, au moins parmi les spécialistes du fait militaire médiéval ${ }^{3}$. L'on sait cependant à quel point les représentations les plus anciennement ancrées peuvent avoir la vie dure, d'autant plus lorsqu'elles ont été véhiculées par des figures tutélaires de l'histoire militaire tel qu'Hans Delbrück qui, il y a moins d'un siècle, écrivait ${ }^{4}$ :

«Pendant un certain temps, l'on croyait même qu'il fallait créditer les Mongols

d'un rôle remarquable dans l'histoire de l'art de la guerre, notamment parce que

des concepts théoriques étaient supposés venir de Tamerlan. Mais en dernière analyse, ils n'accomplirent rien de différent des autres nomades, et les principes de Tamerlan étaient sans réel contenu ».

2 Timothy May va à l'encontre de telles considérations. Mais il ne le fait pas explicitement. Il n'expose d'ailleurs, dans son ouvrage, aucun projet. Un prologue (pp. 1-4) à tonalité essentiellement narrative tient lieu d'introduction. Il y conte la prise de 
Bukhara par Gengis Khan, en 1220, une prise particulièrement violente qui contribua à la diffusion de la légende sanguinaire des Mongols ${ }^{5}$. L'on croit donc comprendre, à la lecture de ce prologue, et plus encore au fil de l'ouvrage, que comme dans sa thèse de doctorat, il se propose de montrer à quel point les Mongols, à rebours donc de l'image qu'ils laissèrent dans la postérité, étaient des hommes de guerre accomplis, dont la pensée militaire et surtout le "système militaire » qu'ils adoptèrent permirent de conquérir et de dominer des contrées si étendues, face à des adversaires si divers. En transformant une armée tribale en une "force militaire régulière opérationnelle », ils créèrent les conditions de leur pérennité6.

3 Dès lors, Timothy May procède par petites touches, en artisan dont on sent bien qu'il a patiemment poli la pièce qu'il façonne avant que de la donner à voir. Sans introduire donc ni conclure son propos - concession sans doute à une volonté de rendre plus facile d'accès un travail originellement académique -, il synthétise l'essentiel des connaissances en matière d'histoire militaire mongole. Le premier chapitre (pp. 5-26), événementiel, retrace à grandes lignes l'histoire de l'empire mongol de 1185 à 1265 . Ensuite, histoire opérationnelle de facture très classique (où stratégie, tactique et art du commandement sont reines) et thématiques chères aux tenants de la New Military History (eux particulièrement sensibles au rôle des armées dans l'évolution des sociétés) se mêlent harmonieusement dans huit chapitres courts et équilibrés: «Recrutement et organisation de l'armée mongole» (chap. 2, pp. 27-41); « Entrainement et équipement du guerrier mongol » (chap. 3, pp. 42-57); « Logistique, approvisionnement et soin médical " (chap. 4, pp. 57-68); "Espionnage, tactiques et stratégie " (chap. 5, pp. 69-85) ; "Leadership » (chap. 6, pp. 86-99); «Opposants des Mongols » (chap. 7, pp. 100-114); « À la guerre avec les Mongols » (chap. 8, pp. 115-137). Enfin, un dernier chapitre s'interroge sur «L'héritage [militaire] des Mongols " (chap. 9, pp. 128-146). Un «Glossaire » (pp. 147-153), une « Sélection bibliographique » (pp. 176-206) bien fournie et un index (pp. 207-214) forment un appareil éditorial honorable. Seuls bémols à déplorer : les cinq cartes hors-texte qui ouvrent le volume sont trop peu lisibles pour être d'une réelle utilité ; une chronologie aurait été bienvenue ; les notes sont reléguées en fin d'ouvrage (pp. 158-175).

4 Selon Timothy May, le principal atout des Mongols fut leur organisation. Il rappelle, après d'autres historiens (en particulier David Morgan), qu'en adoptant une organisation décimale (des unités de 10,100, 1000 et 10000 hommes structuraient l'armée) déjà utilisée par d'autres, Gengis Khan fit non seulement de l'armée un instrument redoutable, mais également la «base d'une structure sociale nouvelle » (p. 27) qui permit de dépasser des subdivisions claniques et tribales inadaptées à la création d'un empire unifié. Les chapitres 3 et 6 permettent de mieux comprendre en quoi l'armée constituait un moteur de développement de puissance sans pareille. Pour le détenteur du pouvoir, elle était aussi un instrument d'ordre et de stabilité dont il lui fallait savoir jouer. D'où la nécessité de faire participer tous ses membres éminents aux réflexions et aux décisions en matière de grande stratégie (cf. pp. 90-91 à propos du quriltai) : «le Khan prenait rarement ses décisions seul » (p. 90). D’où la nécessité, aussi, de choisir avec soin tous ceux destinés à se voir confier des responsabilités ; c'est aussi à ses qualités d'évaluateur que Genghis Khan dut de mettre sur pied une armée efficace: «Au contraire, tout au long de son accession au pouvoir, Genghis Khan fit preuve d'un don extraordinaire pour repérer le talent chez les hommes, qu'ils soient de noble ascendance ou appartiennent au commun» (p. 86). Pour aspirer au 
commandement - à quelque échelle que ce fût -, il fallait prouver sa valeur sur le champ de bataille. Mais être bon stratège, bon tacticien et bon combattant ne suffisait pas: l'on attendait aussi d'un commandant qu'il sût évaluer une situation et susciter l'adhésion de ses hommes, au besoin par l'empathie.

5 Sans doute la mobilité en fut-elle facilitée, dans l'armée - mais sur ce point, Timothy May ne s'étend malheureusement pas. Il ne le fait pas plus sur l'un des problèmes les plus complexes qui soit, pour tous les historiens militaires - celui des effectifs. Il choisit des estimations hautes, et avance des chiffres qui auraient mérité plus ample discussion : les « 540000 hommes, théoriquement » dont Möngke aurait disposé pour conquérir la Chine du Sud (p. 27), au milieu du XIII ${ }^{\mathrm{e}}$ siècle, ne peuvent qu'interpeller. De même, le million d'hommes et plus qu'à leur sommet, les Mongols auraient pu mobiliser, ne doit-il pas être vu comme l'un de ces nombres rhétoriques qui permettent surtout de mieux se représenter leur puissance? Timothy May précise d'ailleurs qu'il faut rapporter ce chiffre à la taille de l'empire mongol, et que « l'on doit se rappeler que ce total n'était et ne pouvait pas être réuni en un seul lieu» (p. 28). Le fait est que les Mongols incorporèrent massivement d'autres nomades et des auxiliaires originaires de terres nouvellement conquises - mais il semble difficile de se faire une idée claire des modalités de cette incorporation.

6 En poursuivant la réflexion sur cette incorporation, l'on peut mieux saisir ce qui faisait la spécificité du "guerrier mongol» (the Mongol warrior) du XIII siècle. Certes suivons l'auteur- il était encore proche des "guerriers des empires steppiques précédents ", mais non sans en être "substantiellement différent ». Cette différence s'explique notamment par la mise en place d'un "système d'entraînement régulier ", système qui permit d'imposer un «sens de la discipline aigu, qui leur conférait une supériorité considérable sur leurs opposants, notamment lors des défaites » (p. 57) : eux n'étaient pas tentés de prendre systématiquement leurs jambes à leur cou et de déguerpir. Faire le lien entre l'entraînement et la discipline, comme le fait Timothy May, permet d'expliquer pourquoi la discipline atteignit, dans les armées mongoles, un «degré inconnu dans les autres armées de l'époque»(André Corvisier)7. Rappelons cependant, comme il le fait lui-même, à quel point les sources sont pauvres, sur ce point. D'ailleurs, nombre de spécialistes (tel John Masson Smith Jr) ne « croient pas que les Mongols étaient bien entraînés» (p. 45). Timothy May se résout alors à s'appuyer sur ce que l'on sait de l'entraînement de "groupes " usant de tactiques similaires aux leurs - les Mamelouks et les Kitan de la Dynastie Liao du nord de la Chine, dont le système militaire, mieux connu, peut servir de "paradigme pour l'étude des Mongols » (p. 43). La méthode, qui a son intérêt, a également ses limites; ainsi, au contraire des Mongols, les Mamelouks étaient sélectionnés, éduqués et entrainés pour la guerre ${ }^{8}$.

7 Les données manquent, également, à propos de l'équipement des Mongols. Étaient-ils ou non bien armés? Timothy May paraît hésiter - il faut dire que les données archéologiques semblent rares, alors que les textes, rédigés dans des langues diverses, n'ont pas, à ma connaissance, fait l'objet d'une exploitation systématique. Sans doute cet armement (offensif et défensif) pouvait-il être de qualité, comme le montrent les quelques pièces reproduites - la photographie d'une paire de bottes du xiiie siècle (Musée d'histoire nationale d'Ulaanbaatar), par exemple, révèle un rembourrage comportant de la cotte de mailles (photographie en bas à gauche de la quatrième page du cahier des illustrations hors-texte ; la technique fait penser à celle du kazāgand des armées proche-orientales ${ }^{9}$. $\mathrm{Vu}$ le nombre de soldats concernés et de campagnes 
pendant lesquelles ils étaient sollicités, cet armement était au moins en partie fourni par le Khan qui, comme en bien d'autres domaines, fit appel aux compétences des peuples passés sous sa domination. Des artisans de toutes contrées (Asie centrale, Perse et Chine) furent ainsi déplacés par centaines; des « colonies d'artisans » (p. 65) peuvent même être repérées.

8 Ainsi, en matière d'armement comme dans tous les domaines de la guerre, le maître mot des Mongols fut le même que celui de toutes les armées médiévales ayant réussi à s'implanter sur de vastes territoires (armées arabes, normandes, croisées etc.): adaptabilité. Une adaptabilité multiforme, qui les conduisit, lorsque cela leur sembla nécessaire, à faire varier leurs stratégies et leurs tactiques les plus usuelles, basées sur la mobilité et la concentration de la puissance de feu (l'efficacité de leurs arcs composites est connue depuis longtemps). Mieux organisés et mieux informés que la plupart de leurs ennemis, passés maîtres dans l'art de la guerre psychologique, ils se convertirent à la guerre de siège, "une fois qu'ils l'eurent comprise » (p. 64) et dès qu'ils purent s'appuyer sur des spécialistes non mongols (tels les Arméniens et les Géorgiens de l'armée qui assiégea Bagdad en 1256, ou les Han et les Coréens qu'ils incorporèrent lors de la guerre contre les Song, au milieu du XIII ${ }^{e}$ siècle). Ils firent dès lors appel à toutes les techniques de siège connues de leur temps, sape et mine, bombardement au moyen de trébuchets d'abord à traction ensuite à contrepoids. Ces trébuchets servaient notamment à projeter du naphte et des "bombes explosives " qu'ils « incorporèrent à leur arsenal dès le début de leur guerre contre les Song " (p. 113 ; cf. aussi p. 104 sur leur utilisation ou non lors des guerres contre les Jin). L'on aurait aimé avoir plus de détails sur cette adoption précoce. De même, trop peu est dit de la flotte créée par les Mongols pour lutter contre les Song ou de l'infanterie sur laquelle ces archers montés durent tout de même s'appuyer - notamment en incorporant des éléments nouvellement soumis. Quel était son poids réel, dans l'armée, et plus généralement au cours des combats ${ }^{10}$ ?

9 Des combats qui, à vrai dire, sont quelque peu négligés par Timothy May, au moins à l'échelle de l'individu : il s'attache surtout au groupe. Il s'efforce certes - avec succès de rendre intelligible nombre d'affrontements, parfois au moyen de croquis très explicites (ainsi, p. 126, ceux de la bataille de la rivière Khalkha, 1224), mais sans réellement s'interroger sur l'expérience humaine que la guerre constituait. À le lire, l'impression qui domine est donc celle d'une guerre sans les hommes, ou plutôt sans émotion, une guerre quelque peu désincarnée car trop schématisée.

10 C'est à ce prix que Timothy May parvient à convaincre de "l'extraordinaire capacité » militaire des Mongols - ce qui ne signifie pas qu'ils formaient des soldats d'élite, loin de là : «dans son ensemble, l'armée mongole avait de bons soldats, mais pas de forces d'élite » (p. 139). Cela leur fut préjudiciable face à de véritables "forces d'élite» tels que les samouraïs japonais ou les Mamelouks - mais, à mon sens, il faut se garder de réduire l'armée mamelouke à ses seuls combattants d'élite.

11 Les Mongols n'en marquèrent pas moins l'histoire de l'art militaire - dans le dernier chapitre, Timothy May souligne, en s'inscrivant dans la voie ouverte par Richard (et non Francis, comme mentionné p. 143 et 144) A. Gabriel ${ }^{11}$, l'importance des Mongols dans l'art militaire. Par-delà leur influence sur les cultures militaires des peuples auxquels ils furent directement ou non confrontés (ainsi les Moscovites, qui adoptèrent une organisation militaire similaire à la leur), ou leur rôle (non véritablement documenté) dans la diffusion de l'arme à feu, ils inspirèrent, au xxe siècle, des 
théoriciens militaires de premier plan comme les britanniques John Frederick C. Fuller (m. 1966) et Basil H. Liddell Hart (m. 1970). A priori adeptes d'une guerre notamment ${ }^{12}$ basée sur la mobilité et le choc, ils considéraient que les tanks étaient peu ou prou les "Mongols modernes" (p. 144, à propos de Fuller). De même, le soviétique Mikhail Nikolayevich Tukhachevsky (m. 1937) voyait les Mongols comme des modèles à suivre. Et l'on peut faire un parallèle entre leur art de la guerre et les réflexions des généraux allemands Hans von Seeckt et Heinz Guderian, qui participèrent activement à la mise en place du style de guerre passé à la postérité sous le nom de blitzkrieg.

On le voit, même si une documentation parfois ténue et le choix de ne pas s'étendre peut laisser le lecteur insatisfait, The Mongol art of War est un ouvrage de qualité, qui pourra tout autant constituer une source d'information utile aux non spécialistes qu'un étai solide à ceux désireux de mener une réflexion plus poussée sur ce qui faisait, n'en déplaise à Hans Delbrück, la spécificité d'une culture de guerre mongole différente de celle des autres peuples nomades et, plus largement, de celle des multiples adversaires qu'ils combattirent et qu'ils vainquirent, qui les défirent aussi, plus rarement certes.

\section{NOTES}

1. Georges Duby, Le Temps des cathédrales : l'art et la société, 980-1420, Paris, Gallimard, 1976, p. 336.

2. The Mechanics of Conquest and Governance: the Rise and Expansion of the Mongol Empire, 1185-1265, PhD Dissertation, Department of History, University of Wisconsin-Madison, 2004.

3. Cf. par exemple, André Corvisier, «Mongols» dans id. (dir.), Dictionnaire d'art et d'histoire militaires, Paris, PUF, 1988, pp. 590-592 ; Richard A. Gabriel et Donald W. Boose, Jr., The Great Battles of Antiquity: A Strategic and Tactical Guide to Great Battles that Shaped the Development of War, Westport, Connecticut et Londres, Greenwood Press, 1994.

4. Hans Delbrück, Geschichte der Kriegskunst im Rahmen der politischen Geschichte, Teil 3, Berlin 1923 ; trad. Walter J. Renfroe, Jr, History of the Art of War, Volume III, Medieval Warfare, Lincoln et Londres, University of Nebraska Press, 1982, n 1 p. 480. Cette note est le seul passage où il est question des Mongols, dans cette somme de 710 pages.

5. Le prologue s'achève d'ailleurs sur l'expression, par le chroniqueur anglais Mathieu Paris (m. 1259), de l'effroi que leur sauvagerie suscitait, en Europe même. La chronique de Mathieu Paris est citée ( $\mathrm{n}^{\circ} 3$ p. 158) dans la traduction de J. A. Giles, vol. I, réimpr. New York, AMS Press Inc., 1968 (1 1 ère éd., 1852), p. 312.

6. Cf. « Remerciements » (h.-t., après la fin de l'ouvrage), à propos de sa thèse : « My examination of how one transforms a tribal levy into a regular operational military force [...] ».

7. « Mongols », Dictionnaire d'art et d'histoire militaires, Paris, PUF, 1988, p. 591.

8. T. May le reconnaît p. 45-46, afin cette fois d'aller contre les arguments avancés par John Masson Smith Jr.

9. Brigandine rembourrée. Cf Abbès Zouache, "L'armement entre Orient et Occident au VI XII ${ }^{\mathrm{e}}$ siècle : Casques, masses d'armes et armures ", AnIsl 41, 2007, pp. 277-326.

10. Voir les avis parfois divergents de David O. Morgan, «Mongols ", EI2 ${ }^{\text {a }}$, t. VII, p. 232 ; id., The Mongols, loc. cit., p. 81 ; Stephen Turnbull et Angus McBride, The Mongols, Oxford, Osprey, 1980, p. 28 («So the Mongol art of warfare provides a lesson by demonstrating that cavalry do not 
necessarily have to rest on a stable infantry base. However, when conditions changed the Mongols had to alter their ideas") ; Pushpa Sharma, " The Military System of the Mongols ", dans Vijai Shankar Srivastava (éd.), Cultural Contours of India. Dr Satyaprakash Felicitation Volume, Humanities Press, Atlantic Highlands, 1981, p. 360 (« Though the Mongol army comprised mainly of cavalry, the infantry was also an important component. Specially the army that came for Indian expedition had large infantry) ; Richard A. Gabriel, Genghis Khan's Greatest General : Subotai the Valiant, Westport, Prager, 2004, p. 28 («For the most part, however, infantry played only a small role in Mongols tactics »)».

11. Richard A. Gabriel, loc. cit., chap. 7 : «The Mongol Military Legacy », pp.127-142. Cet ouvrage, souvent mal informé, a le mérite de s'inscrire dans une véritable perspective d'histoire militaire. Voir le compte-rendu de Timothy May, « Review of Gabriel, Richard A., Genghis Khan's Greatest General: Subotai the Valiant. H-War, H-Net Reviews. January, 2007. URL :http://www.hnet.org/reviews/showrev.php?id=12731 ».

12. Les travaux d'Azar Gat ont montré à quel point il faut se garder de toute simplification, à leur propos : Fascist and Liberal Visions of War : Fuller, Liddell Hart, Douhet and Other Modernists, Oxford, OUP, 1998 ; A History of Military Thought. From the Enlightenment to the Cold War, Oxford, OUP, 2001. 